\title{
Radiographically determined noninvasive adenocarcinoma of the lung: Survival outcomes of Japan Clinical Oncology Group 0201
}

\author{
Hisao Asamura, MD, ${ }^{\mathrm{a}}$ Tomoyuki Hishida, MD, ${ }^{\mathrm{b}}$ Kenji Suzuki, MD, ${ }^{\mathrm{c}}$ Teruaki Koike, MD, ${ }^{\mathrm{d}}$ \\ Kenichi Nakamura, MD, ${ }^{\mathrm{e}}$ Masahiko Kusumoto, MD, ${ }^{\mathrm{a}}$ Kanji Nagai, MD, ${ }^{\mathrm{b}}$ Hirohito Tada, MD, ${ }^{\mathrm{f}}$ \\ Tetsuya Mitsudomi, MD, ${ }^{\mathrm{g}}$ Masahiro Tsuboi, MD, ${ }^{\mathrm{h}}$ Taro Shibata, MSc, ${ }^{\mathrm{e}}$ and Haruhiko Fukuda, MD, ${ }^{\mathrm{e}}$ \\ on behalf of the Japan Clinical Oncology Group Lung Cancer Surgical Study Group
}

\begin{abstract}
Objective: The study objective was to evaluate the long-term survival of patients with radiographically determined noninvasive lung adenocarcinomas.

Methods: A prospective, multi-institutional study on image diagnosis to define early (noninvasive) adenocarcinomas of the lung (Japan Clinical Oncology Group 0201) has shown that a consolidation/tumor ratio on thin-section computed tomography 0.25 or less in cT1a $(\leq 2.0 \mathrm{~cm})$ could be used as a better radiologic criterion for a noninvasive pathology than a consolidation/tumor ratio 0.50 or less in cT1a-b $(\leq 3.0 \mathrm{~cm})$. From the prognostic viewpoints, these criteria were evaluated for 545 patients with adenocarcinoma who underwent lobectomy and lymph node dissection.
\end{abstract}

\begin{abstract}
Results: The subjects consisted of 233 men and 312 women with a median age of 62 years. The median followup period among overall patients was 7.1 years (range, 0-8.5 years). The overall and relapse-free 5-year survivals of the overall patients were $90.6 \%$ and $84.7 \%$, respectively. When a consolidation/tumor ratio 0.5 or less in cT1a-b was used as a cutoff, the 5-year overall survivals of radiologic noninvasive (121 patients, $22.2 \%$ ) and invasive (424 patients, $77.8 \%$ ) adenocarcinomas were $96.7 \%$ and $88.9 \%$, respectively, and the difference was statistically significant $(P<.001, \log$-rank test). With the use of a consolidation/tumor ratio 0.25 or less in cT1a, the 5-year overall survivals of radiologic noninvasive (35 patients, $12.1 \%$ ) and invasive (254 patients, $87.9 \%$ ) adenocarcinomas were $97.1 \%$ and $92.4 \%$, respectively, and the difference was not statistically significant $(P=.259)$.
\end{abstract}

Conclusions: The radiologic criteria of a consolidation/tumor ratio 0.25 or less in cT1a $(\leq 2.0 \mathrm{~cm})$ and 0.50 in cT1a-b $(\leq 3.0 \mathrm{~cm})$ were both able to define a homogeneous group of patients with an excellent prognosis before surgery. (J Thorac Cardiovasc Surg 2013;146:24-30)

Our understanding of the natural history of small, peripheral adenocarcinomas has greatly progressed..$^{1-5}$ This understanding is reflected in the recently revised international multidisciplinary classification of lung adenocarcinoma sponsored by the International Association for the Study of Lung Cancer, American Thoracic Society, and European Respiratory Society. ${ }^{6}$

From the National Cancer Center Hospital, ${ }^{\text {a }}$ Tokyo, Japan; National Cancer Center Hospital East, ${ }^{\mathrm{b}}$ Kashiwa, Japan; Juntendo University Hospital, ${ }^{\mathrm{c}}$ Tokyo, Japan; Niigata Cancer Center Hospital, ${ }^{\mathrm{d}}$ Niigata, Japan; JCOG Data Center, ${ }^{\mathrm{e}}$ National Cancer Center, Tokyo, Japan; Osaka City General Hospital, ${ }^{\mathrm{f}}$ Osaka, Japan; Aichi Cancer Center Hospital, ${ }^{\mathrm{g}}$ Nagoya, Japan; and Kanagawa Cancer Center, ${ }^{\text {h }}$ Yokohama, Japan.

This work was supported in part by National Cancer Center Research and Development Funds (23-A-16, 23-A-18) and Grants-in-Aid for Cancer Research (11S-2, 11S-4, 14S-2, 14S-4, 17S-2, 17S-5, 20S-2, 20S-5) from the Ministry of Health, Labour, and Welfare of Japan.

Disclosures: Authors have nothing to disclose with regard to commercial support.

Received for publication June 19, 2012; revisions received Oct 5, 2012; accepted for publication Dec 11, 2012; available ahead of print Feb 11, 2013

Address for reprints: Hisao Asamura, MD, 5-1-1 Tsukiji, Chuo-ku, Tokyo 104-0045, Japan (E-mail: hasamura@ncc.go.jp).

$0022-5223 / \$ 36.00$

Copyright (c) 2013 by The American Association for Thoracic Surgery

http://dx.doi.org/10.1016/j.jtcvs.2012.12.047
This new classification is characterized by the creation/ abandonment of some terminology for early and advanced adenocarcinomas and by a multidisciplinary approach for the application of the new classification in a clinical setting. In particular, the term "bronchioloalveolar carcinoma" is no longer used, and new concepts have been introduced, such as adenocarcinoma in situ and minimally invasive adenocarcinoma. Invasive adenocarcinomas are classified according to the predominant pattern after comprehensive histologic subtyping with lepidic, acinar, papillary, micropapillary, and solid patterns. The term "mixed subtype adenocarcinoma" is no longer used.

In this new proposal, the surgical features have been described as part of a multidisciplinary approach to the comprehensive classification of adenocarcinomas. Especially for early adenocarcinomas, crucial questions have been raised, such as "Is sublobar (limited) resection adequate oncological treatment for some early adenocarcinomas?" and "Can computed tomography (CT) be used to select patients for sublobar resection?"6,7 The Japan Clinical Oncology Group (JCOG) conducted a prospective, radiologic study of thin-section computed tomography (TSCT) to identify radiologic criteria that predict pathologic noninvasiveness 

Abbreviations and Acronyms
CI = confidence interval
$\mathrm{CT}=$ computed tomography
$\mathrm{C} / \mathrm{T}=$ consolidation/tumor
$\mathrm{GGO}=$ ground-glass opacity
JCOG = Japan Clinical Oncology Group
TSCT $=$ thin-section computed tomography

in clinical IA lung cancer arising in the periphery of the lung (JCOG 0201). ${ }^{8}$ The current study demonstrates the prognosis of the patients who are selected for limited sublobar resection according to the radiologic criteria for noninvasive adenocarcinomas. If we believe that patients with noninvasive adenocarcinomas may be appropriately treated with limited sublobar surgical resection instead of lobectomy, it is crucially important to answer the question, "Can CT be used to select candidates for sublobar resection or those who can benefit from limited sublobar resection?"

In selecting candidates for sublobar resection for early lung adenocarcinomas, however, the preoperative, radiologic prediction of the degree of invasive growth of the tumor has been realized to be a crucial point. Because of the advent of high-resolution CT or TSCT, the radiologic appearance of adenocarcinomas at various stages has been described in detail, and the radiology-pathology correlation has been studied. A localized, nodular lesion characterized by a low-to-moderate increase in CT density that does not obscure lung structures, such as the pulmonary artery/vein and bronchus, is referred to as "ground-glass opacity" (GGO). ${ }^{3}$ It has been shown that GGOs are more likely to be an early form of adenocarcinomas, such as bronchioloalveolar carcinoma, adenocarcinoma in situ, or minimally invasive adenocarcinoma. In particular, some GGOs are accompanied by a solid part, and it has been demonstrated that a solid component represents the portion of invasive growth. These observations suggested that the degree of pathologic invasive growth in adenocarcinoma could be quantified according to the proportion of increased solid density in the CT appearance of the lesion. ${ }^{9}$

On the basis of these observations, the JCOG 0201 study was planned to establish radiologic criteria that could be used to identify pathologic early (noninvasive) adenocarcinomas according to the quantification of the solid component in TSCT. For this purpose, a consolidation/tumor $(\mathrm{C} / \mathrm{T})$ ratio on TSCT was used, and the hypothesis that a $\mathrm{C} / \mathrm{T}$ ratio 0.50 or less in $\mathrm{cT} 1 \mathrm{a}-\mathrm{b}(\leq 3.0 \mathrm{~cm})$ in the TNM staging system (7th) indicates a noninvasive pathology was tested with specificity as the primary end point. The pathology-radiology correlation has been described. $^{8}$ The present study addresses the evaluation of a radiologic criterion in terms of the prognosis after surgery. If we can combine prognostic and pathologic features, we may be able to improve the radiologic criteria for defining noninvasive adenocarcinoma.

\section{PATIENTS AND METHODS \\ The Japan Clinical Oncology Group 0201 Study}

The study design and primary results of the prospective, multiinstitutional study entitled JCOG 0201 have been published. ${ }^{8}$ The study protocol was approved by the JCOG Clinical Trial Review Committee and the institutional review board of each participating center. The study was originally intended to define the radiologic criteria that indicate the pathologic noninvasiveness of adenocarcinomas arising in the periphery of the lung. If the radiologic selection of early adenocarcinomas with an excellent prognosis could be achieved, candidates for limited resection, not for lobectomy, could be precisely identified. Therefore, the radiologic definition of noninvasive adenocarcinoma needs to be assessed not only according to the radiology-pathology correlation but also according to the prognosis. The current study describes the prognosis of patients with resected adenocarcinoma according to different radiologic criteria. In brief, patients who met the following criteria were enrolled in the JCOG 0201 study: (1) a suspected or diagnosed lung cancer based on the findings from a plain x-ray or CT scan; (2) clinical stage IA (ie, T1NOM0) by thoracic enhanced CT; (3) the center of the tumor located peripherally (ie, in the outer half of the lung field) on CT; (4) measurable in at least 1 dimension in TSCT; (5) age 20 to 75 years; (6) no prior thoracotomy; (7) feasible for pulmonary lobectomy; and (8) obtained written informed consent. Before surgery, all patients underwent a contrast-enhanced, thin-section helical CT scan with 1- to 3-mm collimation, with particular focus on the primary tumor to estimate the size of the entire tumor, including GGO and the solid part (consolidation), where the $\mathrm{C} / \mathrm{T}$ ratio was defined as a predictor of pathologic early adenocarcinoma (Figure 1). The patients then underwent surgical resection with at least lobectomy and hilar/mediastinal lymph node dissection. In the JCOG 0201 study, the appropriateness of $\mathrm{C} / \mathrm{T}$ ratios of 0.50 for $\mathrm{cT} 1 \mathrm{a}-\mathrm{b}(\leq 3.0 \mathrm{~cm})$ and 0.25 for $\mathrm{cT} 1 \mathrm{a}(\leq 2.0 \mathrm{~cm})$ was studied by comparing the $\mathrm{C} / \mathrm{T}$ ratio and pathology of the resected specimen. The primary end point of the study was the specificity, which was defined as the proportion of patients with radiologically diagnosed invasive lung cancer determined by the central radiologic review among patients with pathologically diagnosed invasive lung cancer. Pathologic noninvasiveness was defined as pNO disease with neither vascular invasion nor lymphatic permeation on resected specimen.

\section{Patients}

Between December 2002 and May 2004, we enrolled 811 patients from 31 institutions. There were 357 men and 454 women (age range, 27-75 years; median, 61 years). Of these, 562 patients $(69.3 \%)$ underwent major lung resection. After 17 patients who were ineligible on the basis of postoperative pathologic findings were excluded, the prognosis of 545 patients $(67.2 \%)$ was finally studied according to radiologic criteria for noninvasive adenocarcinoma mainly represented by the $\mathrm{C} / \mathrm{T}$ ratio. The subjects of this study are the same as those in the analysis of pathology-radiology correlation of JCOG0201.

\section{Statistical Analysis}

The method of sample size calculation has been described. ${ }^{8}$ Overall survival was defined as the duration from enrollment to death from any cause. For the patients alive, overall survival was censored at the last visit. Relapse-free survival was defined as the duration from enrollment to first recurrence or death from any cause. For the patients alive and recurrence-free, relapse-free survival was censored at the last visit. The probability of survival was estimated by the Kaplan-Meier method, and survival curves were drawn. The difference in survival between the groups was tested by the log-rank test. All $P$ values were 2 -sided. All statistical 


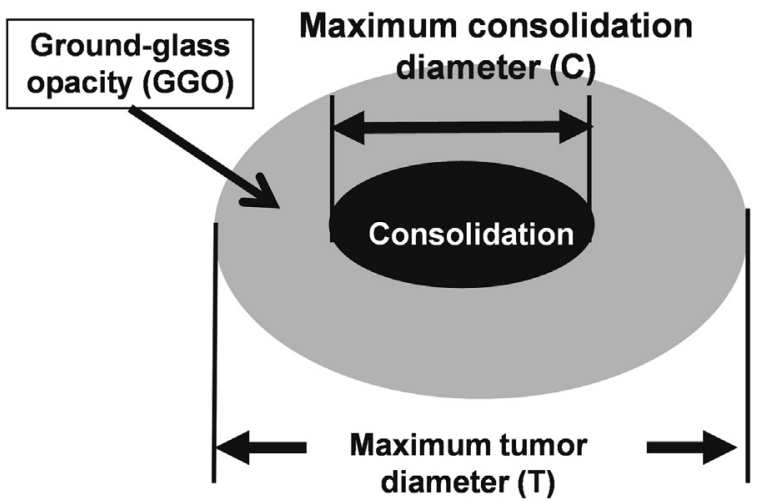

FIGURE 1. Calculation of the $\mathrm{C} / \mathrm{T}$ ratio to define radiologic noninvasive lung cancer on TSCT. The maximum diameter of consolidation $(\mathrm{C})$ is divided by the maximum tumor diameter ( $\mathrm{T}$ ) to give the $\mathrm{C} / \mathrm{T}$ ratio. $G G O$, Ground-glass opacity; $C / T$, consolidation/tumor; TSCT, thin-section computed tomography.

analyses were performed with SAS software release 9.2 (SAS Institute, Inc, Cary, NC) by the JCOG Data Center.

\section{RESULTS}

Prognosis of Patients According to a Consolidation/

Tumor Ratio 0.5 or Less $(n=545)$

The patient characteristics of the entire cT1a-b $(\leq 3.0 \mathrm{~cm})$ population are shown in Table 1. Among the 545 patients, 233 $(42.8 \%)$ were men and $312(57.2 \%)$ were women. The median age was 62 years (range, 35-75 years). The median follow-up period among overall patients was 7.1 years after surgery (range, 0-8.6 years). The overall and relapse-free

TABLE 1. Patient characteristics of the entire cT1a-b $(\leq 3.0 \mathrm{~cm})$ population $(\mathbf{n}=\mathbf{5 4 5})$

\begin{tabular}{lc}
\hline \multicolumn{1}{c}{ Characteristics } & No. of patients $(\%)$ \\
\hline Gender & \\
Men & $233(42.8)$ \\
Women & $312(57.2)$ \\
Age (y) & $62(35-75)$ \\
$\quad$ Median (range) & \\
Maximum tumor diameter on TSCT* & $289(53.0)$ \\
$\leq 2.0 \mathrm{~cm}$ & $254(46.6)$ \\
$>2.0-3.0$ & $2(0.4)$ \\
Unknown & $121(22.2)$ \\
C/T ratio on TSCT* and final pathology $\dagger$ & $115(21.1)$ \\
$\leq 0.5$ (radiologically noninvasive) & $6(1.1)$ \\
$\quad$ Pathologically noninvasive & $424(77.8)$ \\
$\quad$ Pathologically invasive & $263(48.3)$ \\
$>0.5$ (radiologically invasive) & $161(29.5)$ \\
$\quad$ Pathologically noninvasive & \\
$\quad$ Pathologically invasive & \\
\hline TSCT, Thin-section computed tomography; $C / T$, consolidation/tumor. $*$ Maximum tu- \\
mor diameter and a C/T ratio on TSCT were both evaluated by a central radiologic \\
review board. †Patients with adenocarcinoma that was diagnosed at the time of sur- \\
gery were eligible, and 16 patients with a different final pathologic diagnosis were in- \\
cluded in the pathologically invasive group.
\end{tabular}
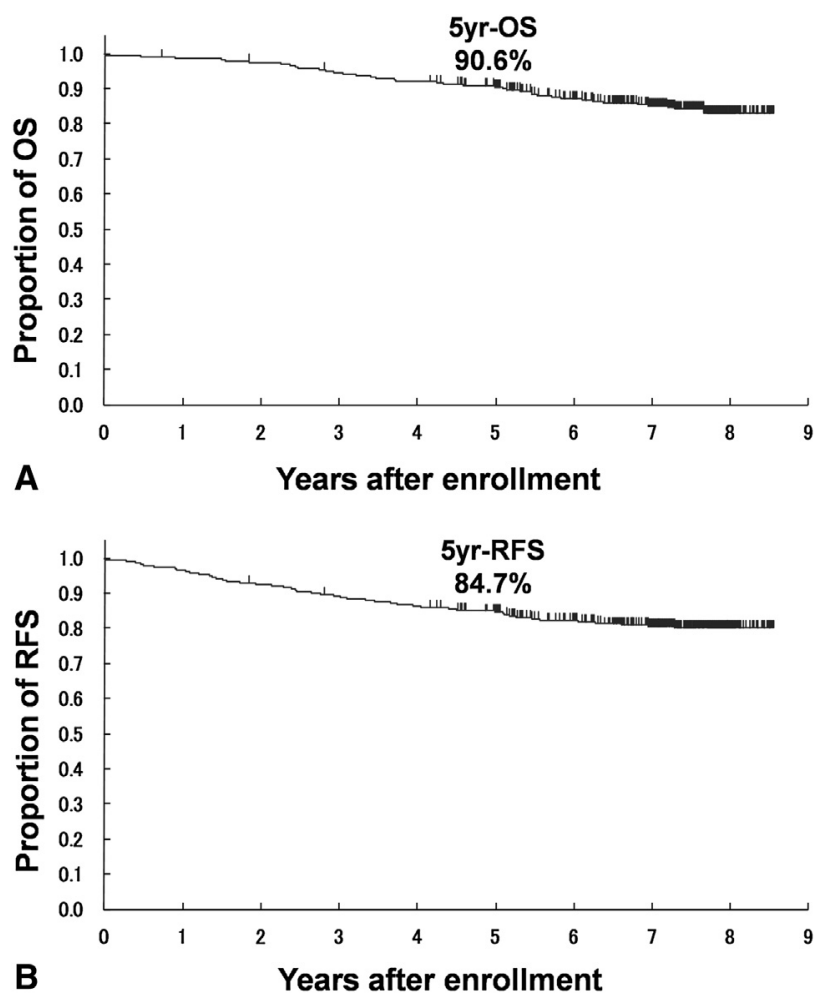

FIGURE 2. Overall (A) and relapse-free (B) survival curves for the entire group (cT1a-b, $\leq 3.0 \mathrm{~cm}, \mathrm{n}=545$ ). $O S$, Overall survival; RFS, relapse-free survival.

survival curves of the cT1a-b $(\leq 3.0 \mathrm{~cm})$ population are presented in Figure 2, and the 5-year overall and relapse-free survivals were $90.6 \%$ and $84.7 \%$, respectively. Among the 545 adenocarcinomas, when a $\mathrm{C} / \mathrm{T}$ ratio 0.5 or less in $\mathrm{cT} 1 \mathrm{a}-\mathrm{b}(\leq 3.0 \mathrm{~cm})$ was used as a radiologic criterion for noninvasive cancer, 121 adenocarcinomas $(22.2 \%)$ were diagnosed as noninvasive and 424 adenocarcinomas (77.8\%) were diagnosed as invasive. Among the 121 radiologic noninvasive adenocarcinomas, $115(95.0 \%)$ were precisely determined to be pathologic noninvasive cancer. Among the 424 radiologic invasive adenocarcinomas, 161 (38.0\%) were precisely determined to be pathologic invasive cancer. Therefore, the specificity and sensitivity were $96.4 \%(95 \%$ confidence interval $[\mathrm{CI}], 92.3-98.7)$ and $30.4 \%(95 \% \mathrm{CI}$, 25.8-35.3), respectively. The overall survival curves for radiologic noninvasive cancer $(\mathrm{n}=121)$ and invasive cancer $(\mathrm{n}=424)$ are presented in Figure 3, A. The 5-year overall survivals for noninvasive cancer and invasive cancer were $96.7 \%$ and $88.9 \%$, respectively, and the difference in overall survival was statistically significant $(P<.001)$. The relapsefree survival curves for radiologic noninvasive cancer $(\mathrm{n}=121)$ and invasive cancer $(\mathrm{n}=424)$ are presented in Figure 3, $B$. The 5-year relapse-free survivals for noninvasive and invasive cancer were $95.9 \%$ and $81.6 \%$, respectively, and the difference in relapse-free survival was statistically significant $(P<.001)$. 

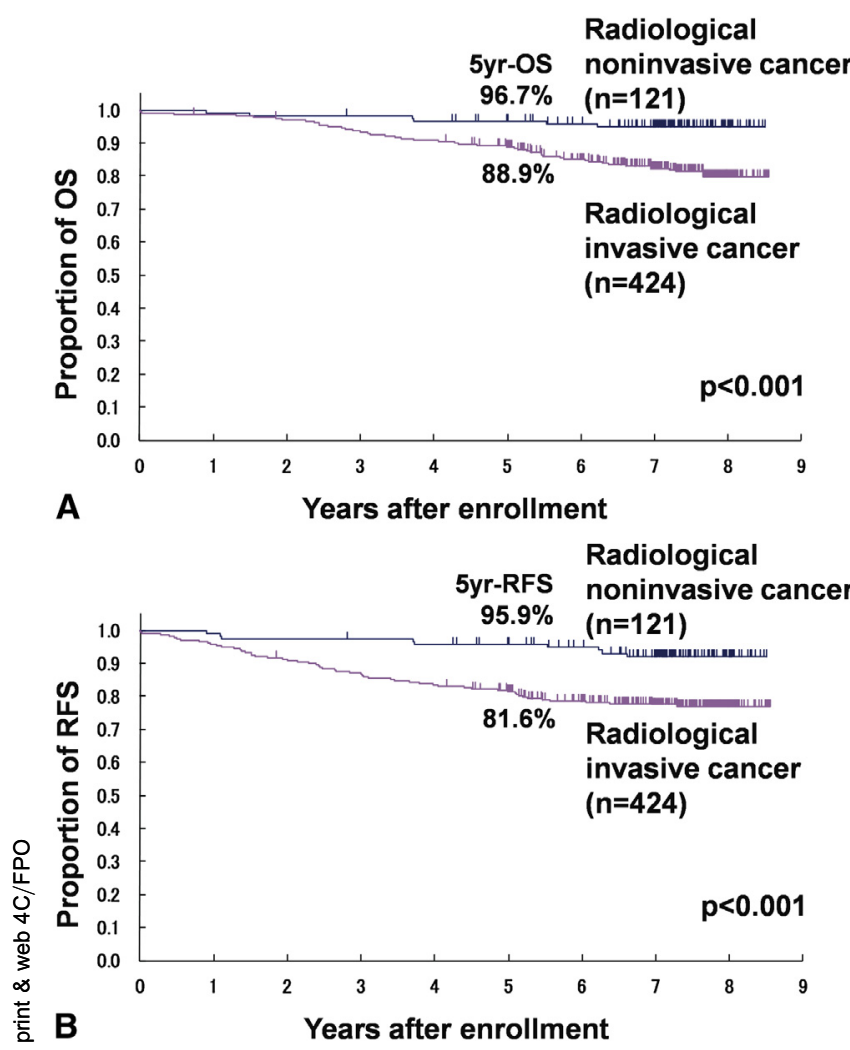

FIGURE 3. Overall (A) and relapse-free (B) survival curves for radiologically noninvasive $(\mathrm{n}=121)$ and invasive $(\mathrm{n}=424)$ adenocarcinomas based on a $\mathrm{C} / \mathrm{T}$ ratio of 0.50 or less in cT1a-b $(\leq 3.0 \mathrm{~cm})$ for noninvasiveness on TSCT. The differences in overall and relapse-free survival are statistically significant $(P<.001$ and $<.001$, respectively). OS, Overall survival; RFS, relapse-free survival; C/T, consolidation/tumor; TSCT, thin-section computed tomography.

\section{Prognosis of Patients According to a Consolidation/ Tumor Ratio 0.25 or Less for cT1a Adenocarcinomas $(\leq \mathbf{2 . 0} \mathbf{~ c m}$ in Size $)(n=289)$}

The patient characteristics of this population are shown in Table 2. Among the 289 patients, 129 (44.6\%) were men and $160(55.4 \%)$ were women. The median age was 61 years (range, 35-75 years). The median follow-up period among overall patients was 7.1 years after surgery (range, $0-8.5$ years). The overall and relapse-free survival curves for the cT1a $(\leq 2.0 \mathrm{~cm})$ population $(\mathrm{n}=289)$ are presented in Figure 4, and the 5-year overall and relapse-free survivals were $93.0 \%$ and $88.9 \%$, respectively. Among the 289 cT1a $(\leq 2.0 \mathrm{~cm})$ adenocarcinomas, when a $\mathrm{C} / \mathrm{T}$ ratio 0.25 or less was used as a radiologic criterion for noninvasive cancer, 35 adenocarcinomas $(12.1 \%)$ were diagnosed as noninvasive and 254 adenocarcinomas $(87.9 \%)$ were diagnosed as invasive. Among the 35 radiologic noninvasive adenocarcinomas, $34(97.1 \%)$ were precisely determined to be pathologic noninvasive cancer. Among the 254 radiologic invasive adenocarcinomas, $78(30.7 \%)$ were precisely determined to be pathologic invasive cancer. Therefore, the
TABLE 2. Patient characteristics of $\mathrm{cT1a}(\leq \mathbf{2 . 0} \mathrm{cm})$ population $(\mathbf{n}=\mathbf{2 8 9})$

\begin{tabular}{lc}
\hline \multicolumn{1}{c}{ Characteristics } & No. of patients $(\%)$ \\
\hline Gender & \\
Men & $129(44.6)$ \\
Women & $160(55.4)$ \\
Age (y) & \\
Median (range) & $61(35-75)$ \\
C/T ratio on TSCT* and final pathology $\dagger$ & \\
$\leq 0.25$ (radiologically noninvasive) & $35(12.1)$ \\
Pathologically noninvasive & $34(11.8)$ \\
Pathologically invasive & $1(0.3)$ \\
$>0.25$ (radiologically invasive) & $254(87.9)$ \\
Pathologically noninvasive & $176(60.9)$ \\
Pathologically invasive & $78(27.0)$ \\
\hline
\end{tabular}

$C / T$, Consolidation/tumor; $T S C T$, thin-section computed tomography. *Maximum tumor diameter and a $\mathrm{C} / \mathrm{T}$ ratio on TSCT were both evaluated by a central radiologic review board. †Patients with adenocarcinoma that was diagnosed at the time of surgery were eligible, and the patients with a different final pathologic diagnosis were included in the pathologically invasive group.

specificity and sensitivity were $98.7 \%$ (95\% CI, 93.2$100.0)$ and $16.2 \%$ (95\% CI, 11.5-21.9), respectively. The overall survival curves for radiologic noninvasive cancer $(\mathrm{n}=35)$ and invasive cancer $(\mathrm{n}=254)$ are presented in Figure 5, $A$. The 5-year overall survivals for noninvasive cancer and invasive cancer were $97.1 \%$ and $92.4 \%$, respectively, and the difference in overall survival was not statistically significant $(P=.259)$. The relapse-free survival curves for radiologic noninvasive cancer $(\mathrm{n}=35)$ and invasive cancer $(\mathrm{n}=254)$ are presented in Figure $5, B$. The 5 -year relapse-free survivals for noninvasive and invasive cancer were $97.1 \%$ and $87.7 \%$, respectively, and the difference in relapse-free survival was not statistically significant $(P=.106)$.

\section{Prognosis of Patients With cT1b Adenocarcinomas $(>\mathbf{2 . 0 - 3 . 0} \mathrm{cm}$ in Size $)(\mathrm{n}=254)$ According to \\ a Consolidation/Tumor Ratio 0.5 or Less}

Two patients with unknown preoperative tumor size were excluded. Among the 254 patients, 104 (40.9\%) were men and $150(59.1 \%)$ were women. The median age was 62 years (range, 37-75 years). The median follow-up period among overall patients was 7.0 years after surgery (range, 0-8.5 years). The overall and relapse-free survivals for the cT1b $(>2.0-3.0 \mathrm{~cm})$ population were $87.8 \%$ and $79.9 \%$, respectively. When a $\mathrm{C} / \mathrm{T}$ ratio 0.5 or less was used as a radiologic criterion for noninvasive cancer, 54 adenocarcinomas $(21.3 \%)$ were diagnosed as noninvasive and 200 adenocarcinomas $(78.7 \%)$ were diagnosed as invasive. The overall survival curves for radiologic noninvasive cancer $(\mathrm{n}=54)$ and invasive cancer $(\mathrm{n}=200)$ are presented in Figure $6, A$. The 5 -year overall survivals for noninvasive cancer and invasive cancer were $96.3 \%$ and $85.5 \%$, respectively, and the difference in overall survival was statistically significant 


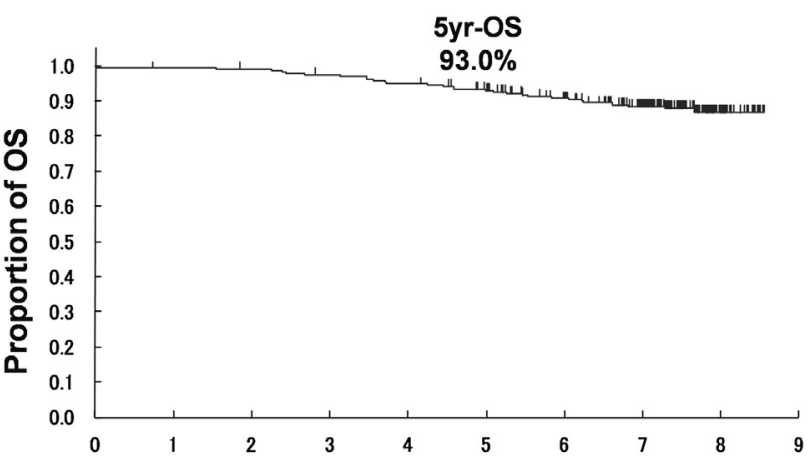

A

Years after enrollment

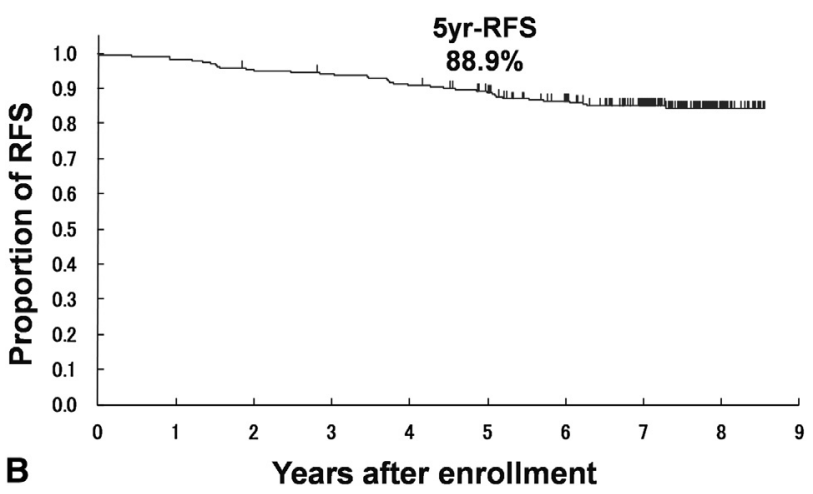

FIGURE 4. Overall (A) and relapse-free (B) survival curves for the cT1a $(\leq 2.0 \mathrm{~cm})$ group $(\mathrm{n}=289)$. OS, Overall survival; RFS, relapse-free survival.

$(P=.003)$. The relapse-free survival curves for radiologic noninvasive cancer $(\mathrm{n}=54)$ and invasive cancer $(\mathrm{n}=200)$ are presented in Figure 6, $B$. The 5-year relapse-free survivals for noninvasive and invasive cancer were $94.4 \%$ and $76.0 \%$, respectively, and the difference in relapse-free survival was statistically significant $(P=.003)$.

\section{DISCUSSION}

During the past 80 years, the surgical mode of pulmonary resection for lung cancer has evolved from pneumonectomy to lobectomy. ${ }^{10,11}$ Currently, resection of the entire tumorbearing lobe is being adopted as the standard mode of surgical resection for lung cancer. During this period, attempts have been made to minimize the resection through lobectomy to limited sublobar resection. Most importantly, the North American Lung Cancer Study Group conducted a prospective, randomized trial that compared limited resection with lobectomy for stage I lung cancer. ${ }^{12}$ The principal finding in that study was a 3 -fold increase in local recurrence $(17.2 \%$ vs $6.4 \%)$ in patients who had sublobar resection and a 2.4-fold increase in those with segmental resection. A $30 \%$ increase in the overall death rate and a $50 \%$ increase in the rate of death with cancer in patients with limited resection were also observed compared with those with lobectomy. Therefore, it was concluded that lobectomy should
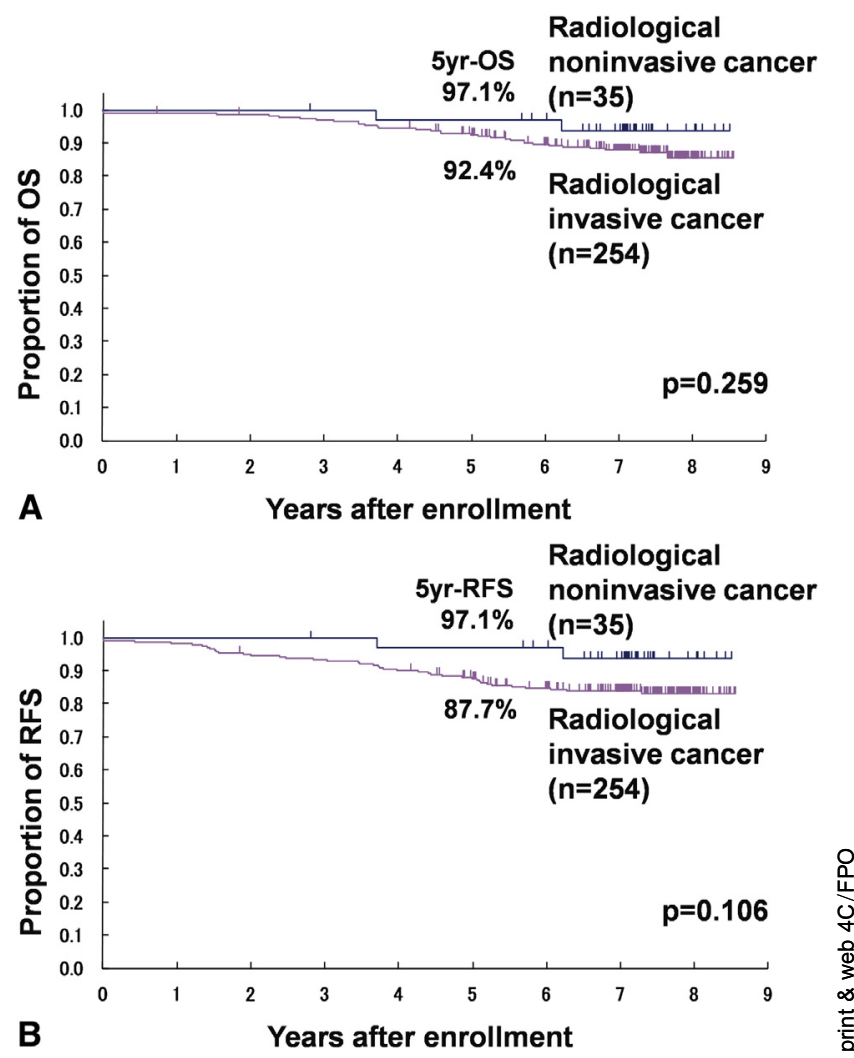

FIGURE 5. Overall (A) and relapse-free (B) survival curves for radiologically noninvasive $(\mathrm{n}=35)$ and invasive $(\mathrm{n}=254)$ adenocarcinomas based on a $\mathrm{C} / \mathrm{T}$ ratio of 0.25 or less in $\mathrm{cT} 1 \mathrm{a}(\leq 2.0 \mathrm{~cm})$ for noninvasiveness on TSCT. The differences in overall and relapse-free survival are not statistically significant $(P=.259$ and .106 , respectively). $O S$, Overall survival; $R F S$, relapse-free survival; $C / T$, consolidation/tumor; $T S C T$, thin-section computed tomography.

still be considered the surgical procedure of choice for peripheral T1N0 non-small cell lung cancer. However, present-day critiques of this study are arising with regard to the marginal prognostic significance, poor preoperative workup for metastasis, slow accrual rate of the study, absence of data on pulmonary function (no demonstration of superiority in pulmonary function for lesser resection), and the notion that this study is outdated.

Despite the results of the North American Lung Cancer Study Group study, many nonrandomized studies have been published, and their results suggested that an equivalent prognosis could be achieved with limited sublobar resection for selected non-small cell lung cancer as with lobectomy. ${ }^{13-18}$ Especially for earlier forms of noninvasive or minimally invasive adenocarcinoma such as bronchioloalveolar carcinoma, it has been shown that these cases present with a GGO appearance on TSCT and could be treated with limited sublobar resections, such as wide wedge resection and segmentectomy. ${ }^{13-18}$ Because of the accumulation of excellent prognoses by limited resection in nonrandomized studies, surgeons require a fair comparison of lobectomy 

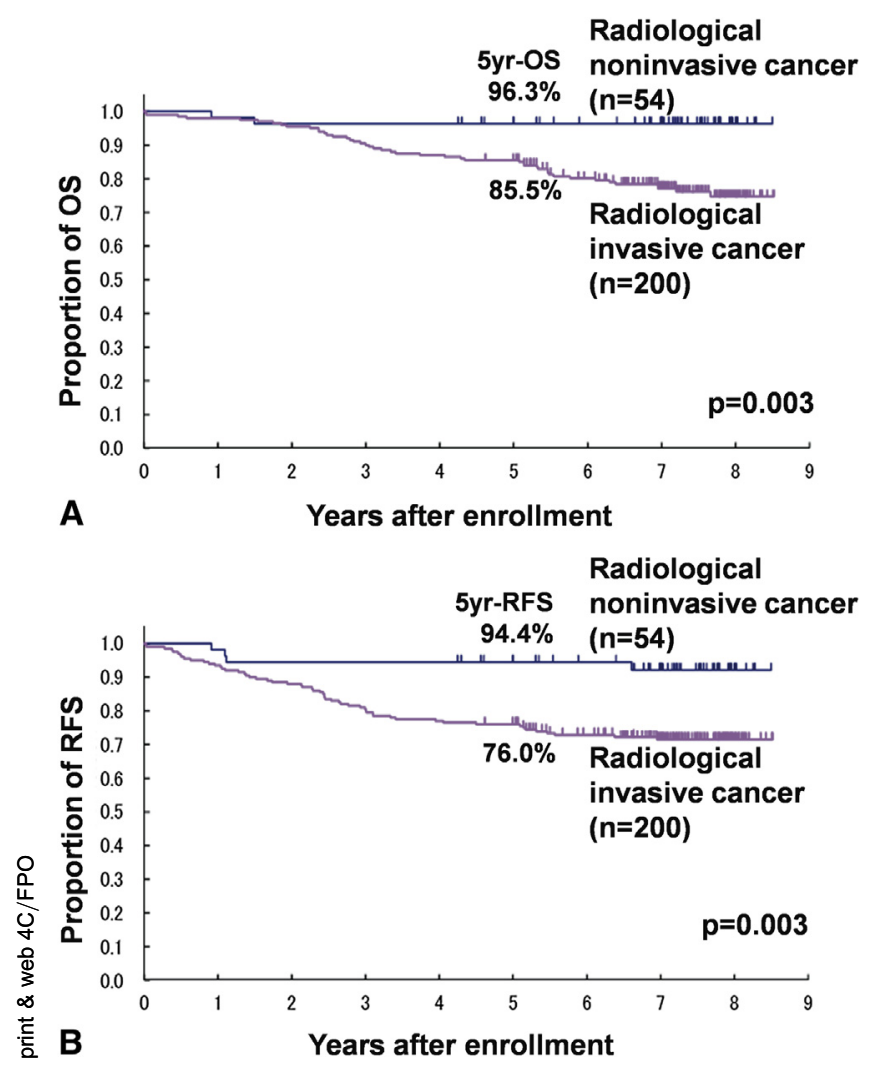

FIGURE 6. Overall (A) and relapse-free (B) survival curves for radiologically noninvasive $(\mathrm{n}=54)$ and invasive $(\mathrm{n}=200)$ adenocarcinomas based on a $\mathrm{C} / \mathrm{T}$ ratio $\leq 0.5$ in $\mathrm{cT} 1 \mathrm{~b}(>2.0-3.0 \mathrm{~cm})$ for noninvasiveness on TSCT. The differences in overall and relapse-free survival are statistically significant $(P=.003$ and .003 , respectively). OS, Overall survival; RFS, relapse-free survival; $C / T$, consolidation/tumor; TSCT, thin-section computed tomography.

and limited resection according to appropriate radiologic criteria for patient selection.

The radiologic criteria for noninvasive adenocarcinoma need to be evaluated by both pathologic and prognostic aspects. The radiology-pathology correlation has been studied in detail in JCOG0201, and the results have been published. ${ }^{8}$ Briefly, JCOG 0201 failed to demonstrate that a $\mathrm{C} / \mathrm{T}$ ratio 0.5 or less in $\mathrm{T} 1 \mathrm{a}-\mathrm{b}$ is precisely predictive of pathologic noninvasiveness. The specificity and sensitivity for these criteria were $96.4 \%(95 \%$ CI, 92.3-98.7) and $30.4 \%$ (95\% CI, 25.8-35.3), respectively, and they did not reach the hypotheses in the JCOG 0201 study that the lower limit for $95 \%$ CI of specificity exceeds $97 \%$. However, according to an exploratory study, a $\mathrm{C} / \mathrm{T}$ ratio 0.25 or less in cT1a $(\leq 2.0 \mathrm{~cm})$ showed a specificity of $98.7 \%$ and a sensitivity of $16.2 \%$, which was more precise for indicating noninvasive pathologic features. On the basis of these results, we recognized that a $\mathrm{C} / \mathrm{T}$ ratio 0.25 or less in cT1a $(\leq 2.0 \mathrm{~cm})$ on TSCT is a better indicator of a noninvasive histology as early adenocarcinoma than a $\mathrm{C} / \mathrm{T}$ ratio 0.50 or less in cT1a-b $(\leq 3.0 \mathrm{~cm})$. In this additional study of JCOG 0201, we looked at the prognoses obtained by these 2 radiologic criteria and focused on whether noninvasive adenocarcinomas defined by these 2 criteria could indicate a good prognosis as early adenocarcinoma.

In contrast to the results regarding the radiologypathology correlation in the JCOG 0201 study, superb prognoses were obtained for noninvasive adenocarcinomas according to both of these criteria: The 5-year overall survivals for a $\mathrm{C} / \mathrm{T}$ ratio 0.50 or less in cT1a-b $(\leq 3.0 \mathrm{~cm})$ and a $\mathrm{C} / \mathrm{T}$ ratio 0.25 or less in $\mathrm{cT} 1 \mathrm{a}(\leq 2.0 \mathrm{~cm})$ were $96.7 \%$ and $97.1 \%$, respectively. Although our previous report stated that a $\mathrm{C} / \mathrm{T}$ ratio 0.25 or less in $\mathrm{cT} 1 \mathrm{a}(\leq 2.0 \mathrm{~cm})$ was an appropriate indicator to select patients with noninvasive lung adenocarcinomas suitable for limited sublobar resection, ${ }^{8}$ these results indicate that even with a generous radiologic criterion on $\mathrm{TSCT}$ (a $\mathrm{C} / \mathrm{T}$ ratio $\leq 0.50$ in cT1a-b), we can precisely select a group of patients with early adenocarcinomas who have an excellent prognosis. For these patients, it is considered that limited sublobar resection achieves a prognosis that is almost the same as that with lobectomy and a better preservation of the lung parenchyma. In terms of the postoperative prognosis, this analysis indicates that noninvasive adenocarcinomas can be properly selected on TSCT using radiologic criteria of a $\mathrm{C} / \mathrm{T}$ ratio of 0.50 or 0.25 .

There is a caution regarding the excellent prognoses of the patients with noninvasive adenocarcinomas in this study. Patients included in the JCOG 0201 study underwent lobectomy and hilar/mediastinal lymph node dissection, which is usually indicated for any type of resectable lung cancer as a radical resection. The prognoses in this study were achieved by such radical resection; therefore, the same prognoses are not warranted for these tumors if they are resected with limited sublobar resections, although this was suggested by previous studies with case series. ${ }^{13-18}$ We should still be prudent in addressing the excellent prognoses of early adenocarcinomas with surgical resection other than lobectomy.

On the basis of these results, 2 prospective, collaborative studies between JCOG and the West Japan Oncology Group, JCOG0804/WJOG4507L (UMIN-CTR [www. umin.ac.jp/ctr/] No. UMIN000002008) and JCOG0802/ WJOG4607L (UMIN-CTR No.UMIN000002317), are under way on peripherally located adenocarcinomas of the lung. ${ }^{19}$ In these studies, a radiologic criterion of a $\mathrm{C} / \mathrm{T}$ ratio 0.25 or less in cT1a is used to define noninvasive adenocarcinomas. For radiologic noninvasive adenocarcinomas with a tumor diameter of $2 \mathrm{~cm}$ or less, a phase II study with $1 \mathrm{ex}$ perimental arm (JCOG0804/WJOG4507L) is under way in which tumors are resected with a wide wedge resection. As of March 2012, the target number of accrual (330 patients) was reached, and maturation of follow-up data on recurrence and prognosis is awaited. For radiologic invasive adenocarcinomas with a tumor diameter $2.0 \mathrm{~cm}$ or less and 
a $\mathrm{C} / \mathrm{T}$ ratio greater than 0.25 , a prospective, randomized phase III study (JCOG0802/WJOG4607L) between lobectomy and segmentectomy in a noninferiority setting is under way. The primary and key secondary end points are overall survival and postoperative pulmonary function, respectively. If the prognosis of those undergoing segmentectomy is not inferior to that of those undergoing lobectomy and the pulmonary function of those undergoing segmentectomy is significantly better than that of those undergoing lobectomy, we will conclude that segmentectomy should be the standard mode of pulmonary resection for a peripherally located radiologic invasive adenocarcinoma with a diameter of $2.0 \mathrm{~cm}$ or less and a C/T ratio greater than 0.25 . The target number of patients is 1100 , and accrual is under way. This study will clarify the pathologic and survival outcomes of the patients with cT1a $(\leq 2.0 \mathrm{~cm})$ tumor and a $\mathrm{C} / \mathrm{T}$ ratio ranging from 0.25 to 0.5 , and might validate the radiologic definition of a $\mathrm{C} / \mathrm{T}$ ratio 0.5 or less for noninvasive lung adenocarcinoma among the $\mathrm{cT} 1 \mathrm{a}(\leq 2.0 \mathrm{~cm})$ group. In North America, a similar study entitled Cancer and Leukemia Group B 140503 is also under way, in which the prognosis and preservation of pulmonary function is compared between lobectomy and limited resection in a noninferiority setting. ${ }^{20,21}$ The current survival analysis showed that noninvasive adenocarcinomas indicative of limited resection could be selected by the size of $3 \mathrm{~cm}$ or less and a $\mathrm{C} / \mathrm{T}$ ratio 0.5 or less. Even the patients with $\mathrm{cT} 1 \mathrm{~b}$ tumors $(>2-3 \mathrm{~cm})$ may be candidates for limited resection if their preoperative $\mathrm{C} / \mathrm{T}$ ratio is 0.5 or less. For cT1b tumors, segmentectomy but not wide wedge resection should be considered as an appropriate limited resection to obtain adequate surgical margins. Future prospective study will be needed to investigate the clinical significance of intentional segmentectomy for such a population.

\section{CONCLUSIONS}

Despite the finding that a noninvasive pathology is better predicted with a C/T ratio 0.25 or less on TSCT in cT1a $(\leq 2.0 \mathrm{~cm})$ than with 0.50 or less in cT1a-b $(\leq 3.0 \mathrm{~cm})$, both of these radiologic criteria could identify a group of patients with an excellent prognosis, with a 5-year overall survival of approximately $97 \%$. These criteria can be used to select patients with peripherally located adenocarcinomas in whom a limited resection, such as wide wedge resection or segmentectomy, might be safely indicated.

The authors thank Mieko Imai, BSc, and Tomohisa Furuya, BSc, for support with data management, and Junki Mizusawa, $\mathrm{MSc}$, for the statistical analysis.

\section{References}

1. Noguchi M, Morikawa A, Kawasaki M, Matsuno Y, Yamada T, Hirohashi S, et al. Small adenocarcinoma of the lung. Histologic characteristics and prognosis. Cancer. 1995;75:2844-52.

2. Koike T, Terashima M, Takizawa T, Watanabe T, Kurita Y, Yokoyama A. Clinical analysis of small-sized peripheral lung cancer. J Thorac Cardiovasc Surg. 1998; 115:1015-20.

3. Suzuki K, Asamura H, Kusumoto M, Kondo H, Tsuchiya R. "Early" peripheral lung cancer: prognostic significance of ground glass opacity on thin-section computed tomographic scan. Ann Thorac Surg. 2002;74:1635-9.

4. Suzuki K, Kusumoto M, Watanabe S, Tsuchiya R, Asamura H. Radiologic classification of small adenocarcinoma of the lung: radiologic-pathologic correlation and its prognostic impact. Ann Thorac Surg. 2006;81:413-9.

5. Travis WD, Garg K, Franklin WA, Wistuba II, Sabloff B, Noguchi M, et al. Evolving concepts in the pathology and computed tomography imaging of lung adenocarcinoma and bronchioloalveolar carcinoma. J Clin Oncol. 2005; 23:3279-87.

6. Travis WD, Brambilla E, Noguchi M, Nicholson A, Geisinger K, Yatabe Y, et al. International Association for the Study of Lung Cancer/American Thoracic Society/European Respiratory Society international multidisciplinary classification of lung adenocarcinoma. J Thorac Oncol. 2011;6:144-85.

7. Van Schil P, Asamura H, Rusch VW, Mitsudomi T, Tsuboi M, Brambilla E, et al. Surgical implications of the new IASLC/ATS/ERS adenocarcinoma classification. Eur Respir J. 2012;39:478-86.

8. Suzuki K, Koike T, Asakawa T, Kusumoto M, Asamura H, Nagai K, et al. A prospective radiological study of thin-section computed tomography to predict pathological noninvasiveness in peripheral clinical IA lung cancer (Japan Clinical Oncology Group 0201). J Thorac Oncol. 2011;6:751-6.

9. Kodama K, Higashiyama M, Yokouchi H, Takami K, Kuriyama K, Mano M, et al. Prognostic value of ground-glass opacity found in small lung adenocarcinoma on high-resolution CT scanning. Lung Cancer. 2001;33:17-25.

10. Cahan WG, Watson WL, Pool JL. Radical pneumonectomy. J Thorac Surg. 1951; 22:449-73.

11. Cahan WG. Radical lobectomy. J Thorac Cardiovasc Surg. 1960;39:555-72.

12. Ginsberg RJ, Rubinstein LV. Randomized trial of lobectomy versus limited resection for T1 N0 non-small cell lung cancer. Lung Cancer Study Group. Ann Thorac Surg. 1995;60:615-22.

13. Watanabe S, Watanabe T, Arai K, Kasai T, Haratake J, Urayama H. Results of wedge resection for focal bronchioloalveolar carcinoma showing pure groundglass attenuation on computed tomography. Ann Thorac Surg. 2002;73: 1071-5.

14. Yoshida J, Nagai K, Yokose T, Nishimura M, Kakinuma R, Ohmatsu H, et al. Limited resection trial for pulmonary ground-glass opacity nodules: fifty-case experience. J Thorac Cardiovasc Surg. 2005;129:991-6.

15. Okada M, Koike T, Higashiyama M, Yamato Y, Kodama K, Tsubota N. Radical sublobar resection for small-sized non-small cell lung cancer: a multicenter study. J Thorac Cardiovasc Surg. 2006;132:769-75.

16. El-Sherif A, Gooding WE, Santos R, Pettiford B, Ferson PF, Fernando HC, et al. Outcome of sublobar resection versus lobectomy for stage I non-small cell lung cancer: a 13-year analysis. Ann Thorac Surg. 2006;82:408-16.

17. Schuchert MJ, Pettiford BL, Keeley S, D'Amato TA, Kilic A, Close J, et al. Anatomic segmentectomy in the treatment of stage I non-small cell lung cancer. Ann Thorac Surg. 2007;84:926-33.

18. Wolf AS, Richards WG, Jaklitsch MT, Gill R, Chirieac LR, Colson YL, et al. Lobectomy versus sublobar resection for small ( $2 \mathrm{~cm}$ or less) non-small cell lung cancers. Ann Thorac Surg. 2011;92:1819-23.

19. Nakamura K, Saji H, Nakajima R, Okada M, Asamura H, Shibata T, et al. A phase III randomized trial of lobectomy versus limited resection for small-sized peripheral non-small cell lung cancer (JCOG0802/WJOG4607L). Jpn J Clin Oncol. 2010;40:271-4.

20. Swanson SJ. Video-assisted thoracic surgery segmentectomy: the future of surgery for lung cancer? Ann Thorac Surg. 2010;89:S2096-7.

21. Blasberg JD, Pass HI, Donington JS. Sublobar resection: a movement from the Lung Cancer Study Group. J Thorac Oncol. 2010;5:1583-93. 